\title{
Density of States in Graphene with Vacancies: Midgap Power Law and Frozen Multifractality
}

\author{
V. Häfner, ${ }^{1,2}$ J. Schindler, ${ }^{1,2}$ N. Weik, ${ }^{1,2}$ T. Mayer, ${ }^{1,2}$ S. Balakrishnan, ${ }^{3}$ R. Narayanan, ${ }^{3}$ S. Bera, ${ }^{4,}$ and F. Evers ${ }^{1,2,5}$ \\ ${ }^{1}$ Institute of Nanotechnology, Karlsruhe Institute of Technology, Campus North, D-76344 Karlsruhe, Germany \\ ${ }^{2}$ Institut für Theorie der Kondensierten Materie, Karlsruhe Institute of Technology, Campus South, D-76128 Karlsruhe, Germany \\ ${ }^{3}$ Department of Physics, Indian Institute of Technology Madras, Chennai 600036, India \\ ${ }^{4}$ Institute Néel and Université Grenoble Alpes, F-38042 Grenoble, France \\ ${ }^{5}$ Center of Functional Nanostructures, Karlsruhe Institute of Technology, Campus South, D-76131 Karlsruhe, Germany
}

(Received 30 April 2014; published 29 October 2014)

\begin{abstract}
The density of states $Q(E)$ of graphene is investigated numerically and within the self-consistent $T$-matrix approximation in the presence of vacancies within the tight binding model. The focus is on compensated disorder, where the concentration of vacancies $n_{A}$ and $n_{B}$ in both sublattices is the same. Formally, this model belongs to the chiral symmetry class BDI. The nonlinear sigma model predicts for BDI a Gade-type singularity $\varrho(E) \sim|E|^{-1} \exp \left[-|\log (E)|^{-1 / x}\right]$. Our numerical data are comparable to this result in a preasymptotic regime that gives way, however, at even lower energies to $\varrho(E) \sim E^{-1}|\log (E)|^{-\tilde{x}}$, $1 \leq \tilde{x}<2$. We take this finding as evidence that, similar to the case of dirty $d$-wave superconductors, generic bipartite random hopping models may also exhibit unconventional (strong-coupling) fixed points for certain kinds of randomly placed scatterers if these are strong enough. Our research suggests that graphene with (effective) vacancy disorder is a physical representative of such systems.
\end{abstract}

DOI: 10.1103/PhysRevLett.113.186802

PACS numbers: 73.22.Pr, 71.23.-k, 72.80.Vp

Graphene is a hot topic in material sciences and condensed-matter physics [1]. The material is interesting because its electronic structure hosts two Dirac cones. Since only the $\pi_{z}$ orbitals make significant contributions to the relativistic sectors of the band structure, a tight-binding description of the material is frequently employed that keeps a single orbital per carbon atom and only nearestneighbor hopping. Within this description, it is easy to see that disorder introduced by a random distribution of vacancies has nontrivial effects. For instance, it is well known that a single impurity populates a midgap state that is power-law localized [2,3]. With a finite concentration of vacancies a rich plethora of new phenomena emerges. One distinguishes the "compensated" case, the same concentration of vacancies in each sublattice $\bar{n}=n_{A}=n_{B}$, from the uncompensated case, $n_{A}>n_{B}$. In the latter case, one expects that the density of states (DoS) exhibits a (pseudo-) gap, while for compensated disorder a sharp peak is observed [1]. Most studies focus on the balanced case at concentrations well below the percolation threshold, $\bar{n} \lesssim 30 \%$. At present, only very few aspects have been investigated in detail, despite the importance of the DoS for transport and optical properties of the functionalized material $[4,5]$.

Graphene with vacancies represents a bipartite random hopping system with time reversal and spin rotational invariance. Following the Zirnbauer-Altland classification of disordered metals, it belongs to symmetry class BDI, $[6,7]$. In the presence of weak bond disorder, the nonlinear $\sigma$ model predicts for the DoS

$$
\ln |E \varrho(E)| \sim-|\ln (E / \mathfrak{D})|^{1 / x}, \quad|E| \lesssim \mathfrak{D},
$$

where $\mathfrak{D}(\bar{n})$ denotes a microscopic energy scale. [7] The exponent $1 / x$ reflects a peculiar feature of the renormalization group (RG) flow found by Gade and Wegner in a perturbative RG study [8,9]. Their analysis shows that the energy flow with the RG scale $L$ is $|\ln \epsilon| \propto z(L)|\ln L|$. Unlike the case with conventional critical behavior, the dynamical exponent $z$ is not a constant here but rather $z(L) \propto \ln L$, so $|\ln \epsilon| \propto|\ln L|^{2}$, and correspondingly an exponent $x=2$ was obtained [10].

Later it was argued that the logarithmically growing exponent $z$ is an indication of "freezing" $[12,13]$. Freezing sets in when disorder has become so strong that critical wave functions concentrate in rare regions of the sample with very weak, power-law tails leaking out of these "optimal" domains. In such situations, observables that derive from moments of wave function amplitudes higher than the first one cease to be sensitive to the sample geometry so that their "flow" with the system size is "frozen." Freezing implies that at $z \geq 3$ rare events dominate the energy scaling and a new dependency $z \approx$ $4 \sqrt{\ln L}-1$ sets in [7]. As a consequence, the Gade exponent $x=2$ gives way to $x=3 / 2$ and the zero-energy singularity becomes slightly weaker in the frozen limit.

A strong increase of the DoS near zero energy has been observed in several numerical works [3,5,14-16], but a quantitative check of the prediction, Eq. (1), is still missing. Here, we present such an analysis. We confirm the existence of a parametrically wide energy window where 
$Q(E)$ indeed follows Eq. (1). However, at very-low energies, Eq. (1) is not valid. Instead, the DoS crosses over to new behavior with a significantly stronger singularity, $1 /[E|\log (E)| \tilde{x}]$, with $2>\tilde{x} \geq 1$.

Model and methods.-Graphene exhibits two distinct scaling regimes for the DoS. In one regime, the linear dependence on energy rules; it is accessible via perturbation theory employing the self-consistent $T$-matrix approximation (SCTMA). At lower energies, the DoS exhibits the upturn that is the focus of this Letter and poses significant numerical challenges. For instance, it is not directly accessible to exact diagonalization methods because the DoS requires one to treat a very large number of eigenvalues $\left(\sim \bar{n} L^{2}\right)$ for matrices of dimension exceeding $L^{2} \sim 10^{7}$.

SCTMA: The SCTMA was applied to graphene before $[4,17]$ and also to $d$-wave systems (see e.g., Refs. [18,19]); here, we use it to calculate the DoS.

Stochastic time evolution: The SCTMA results are then compared against numerical simulation data for $Q(E)$ as obtained from a tight-binding Hamiltonian of the honeycomb lattice $\hat{H}=-t \sum_{\langle i j\rangle} c_{i}^{\dagger} c_{j}$ where as usual $\langle i j\rangle$ indicates nearest-neighbor hopping. The disorder average is performed at vacancy concentration $\bar{n}$ fixed and the same for both sublattices. We employ a numerical technique similar to Ref. [14] exploiting $\varrho(E)=\int_{-\infty}^{\infty} d \tau \varrho(\tau) e^{\mathrm{i} E \tau}$ with the exact stochastic representation

$$
\varrho(\tau)=\frac{1}{2 \pi} \overline{\{\langle\phi|\exp (-\mathfrak{i} \hat{H} \tau)| \phi\rangle\}_{\text {in }}} .
$$

Here, $|\phi\rangle$ represents a random initial state, and $\{\ldots\}_{\text {in }}$ denotes an ensemble average of such states. For the evaluation of the matrix element, we employ a standard Krylov-subspace approach, with a conservative choice of the width of the integration steps, typically $d \tau=0.01$ (units: $t^{-1}$ ), and an observation window of $10^{6}$ steps corresponding to a time $T_{\mathrm{obs}}=10^{4}$ [20]. In order not to lose correlations due to methodological artifacts over the observation time, the time increment $d \tau$ should become significantly smaller with growing $T_{\mathrm{obs}}$. As it turns out, this makes the stochastic time evolution numerically highly demanding at very-low energies and unpractical at energies significantly below $10^{-3} t$.

Generalized multifractal analysis: In order to explore $\varrho(E)$ at very-low energies, we first calculate the localization length $\xi(E)$ with spectral methods and then invoke a plausible relation between $\xi(E)$ and $\varrho(E)$. If one assumes that a localization volume $\xi^{2}$ has typically one state with lowest energy $E_{\xi}$ one has $\left|E_{\xi}\right| \xi^{2} \varrho\left(E_{\xi}\right)=\mathcal{O}(1)$. Hence, $\xi(E) \approx|E Q(E)|^{-1 / 2}$. The expression is familiar from the standard weak coupling analysis [7]. A complication arises because the same analysis predicts the form of Eq. (1) for the DoS that turns out to be incompatible with our numerical data-as we already mentioned. Hence, a more general form $\left|E_{\xi}\right| \xi^{2} \varrho\left(E_{\xi}\right)=r(\ell / \xi)$ should be considered
( $\ell$ : a microscopic length). Partially inspired by most recent analytical work [23], we argue in the Supplemental Material [24] that a reasonable assumption would be $r(X) \approx 1 / X^{\tilde{y}}$ (with $\tilde{y}=1$ ) at energies not too low so that

$$
\xi(E) \approx|E \varrho(E)|^{-1 /(2+\tilde{y})} .
$$

Similar to Ref. [31], we employ a generalized multifractal analysis to extract the localization length even at very-low energies. This analysis is motivated in the present context from the fact that multifractality at the Dirac point is a topic of interest per se. The central observable is the inverse participation ratio (IPR), $P_{q}(E)=\int_{L^{2}} d \mathbf{r}\left|\psi_{m}(\mathbf{r})\right|^{2 q}$. (For numerical efficiency, we average over a small number of states with energies $\epsilon_{m}$ inside an interval about $E$. In addition, we also perform a disorder average at fixed $\bar{n}$ that we indicate via $\bar{P}_{q}$.) To address the localization length $\xi(E)$, one works at finite energies $|E|>0$ in the vicinity of the critical fixed point where a scaling ansatz

$$
\overline{P_{q}}=L^{-\tau_{q}} \mathcal{F}_{q}[L / \xi(E)]
$$

applies. We will extract $\xi(E)$ by scaling our numerical data according to this relation. The wave function data have been obtained in a well documented way (e.g., Refs. [32,33]), employing standard sparse matrix routines [34].

Results.-SCTMA: The DoS as obtained from the self-consistency cycle of the SCTMA is shown in Fig. 1 with dashed lines. In the limit of large and low energies, we recover the expected qualitative behavior: If the energy exceeds a characteristic scale set by $\Delta(\bar{n})=$ $v_{F} \sqrt{\pi \bar{n} / \ln \left(n^{*} / \bar{n}\right)}$, with $n^{*}=W^{2} / \pi v_{F}^{2}$, and $W$ a highenergy cutoff [17], the DoS essentially remains unaffected by the impurities, thus retaining the characteristic linear form reminiscent of clean graphene at high energies. (Our data suggests $n^{*} \approx 1$.) However, in the low-energy limit

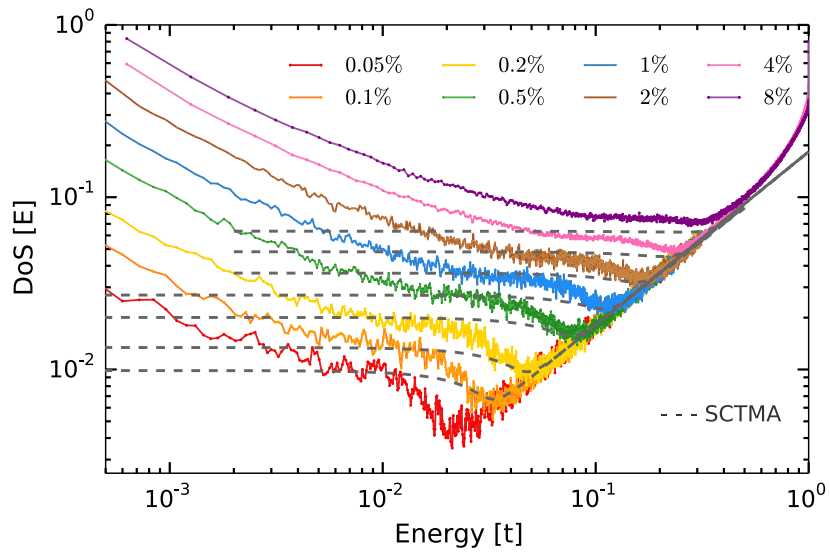

FIG. 1 (color online). Density of states of graphene with $\bar{n}=0.1 \%-8 \%$ vacancies in either sublattice. Comparison of SCTMA and tight-binding simulation. 
$E \ll \Delta(\bar{n})$, the Dirac singularity broadens and one obtains a constant value for the DoS.

Tight-binding-simulation: energy: Since the SCTMA ignores multiple scattering at two (or more) impurity configurations, quantum-interference processes are absent. Hence, within the SCTMA one does not expect any indication of the $E^{-1}$ singularity predicted in Eq. (1). To investigate this, we resort to a numerical simulation of the DoS in the lattice model. As one might have suspected, the characteristic minimum seen within the SCTMA (discussed in the Supplemental Material [24]) is reproduced in the lattice simulation of Fig. 1 and turns out to be even more pronounced there. Quantum interference becomes important at energies below a scale $\mathfrak{D}(\bar{n})$ where it gradually enhances the (upturning) curvature.

Tight-binding-simulation: time: At lowest energies, the Fourier transformation exhibits a sensitivity to the window of integration times. Even though artifacts are generally weak, for the present purpose we will work in the time representation of observation time $T_{\mathrm{obs}}=10^{4}$. Figure 2 displays the first out of the two key results of this work: at intermediate times the DoS takes a form consistent with Eq. (1)

$$
\varrho(\tau) \approx \bar{n} A_{0} \exp \left[-\mathfrak{a}_{0}\left|\ln \left(\tau / \tau_{0}\right)\right|^{1 / x}\right], \quad \mathfrak{D}^{-1} \ll \tau \ll \tau_{\bar{n}}^{*} .
$$

The crossover scale $\tau_{\bar{n}}^{*}$ is very rapidly decreasing if $\bar{n}$ grows from $3 \%$ to $10 \%$. As a consequence, the onset of the verylong time regime can be investigated with the time propagation method. As shown in Fig. 3, at times exceeding $\tau_{\bar{n}}^{*}$ the decay of $\varrho(\tau)$ is much slower even than $1 / \ln (\tau)$. The accessible time window is too small in order to reliably discriminate possible cases, $1 \leq \tilde{x}<2$,

$$
\varrho(\tau)=\bar{n} A_{\bar{n}}\left|\ln \left(\tau / \tau_{\bar{n}}+\mathfrak{a}_{\bar{n}}\right)\right|^{-\tilde{x}+1}, \quad \tau_{\bar{n}}^{*} \ll \tau .
$$

[Even $\tilde{x} \rightarrow 1$, i.e., $\varrho(\tau)=\bar{n} A_{\bar{n}} /\left\{\ln \left[\ln \left(\tau / \tau_{\bar{n}}\right)\right]+\mathfrak{a}_{\bar{n}}\right\}$, would not be incompatible with the data (see Figs. 2 and 3).] What can safely be concluded at this point is that at very-low energies $|E| \varrho(E) \propto 1 / \ln (|E|)^{\tilde{x}}, \quad 1 \leq \tilde{x}<2$ at variance with Eq. (1).

Generalized multifractal analysis: We have calculated the IPR near four different energies covering the range $10^{-3} t-10^{-7} t$. The resulting master curve $\mathcal{F}$ defined in Eq. (4) is displayed in Fig. 4. In the regime of large system sizes $L \gg \xi(E)$ all curves exhibit a plateau indicating that the IPR is independent of the growing system size: we observe the insulating behavior expected for the AI class that eventually governs all energies except $E=0$. At smaller $L / \xi(E)$ values, a power-law regime begins to develop that governs intermediate system sizes but is cut off at smallest values $L \ll \xi(E)$ where the slope begins to decrease again. This peculiar feature foreshadows the behavior at the critical fixed point. We believe that it

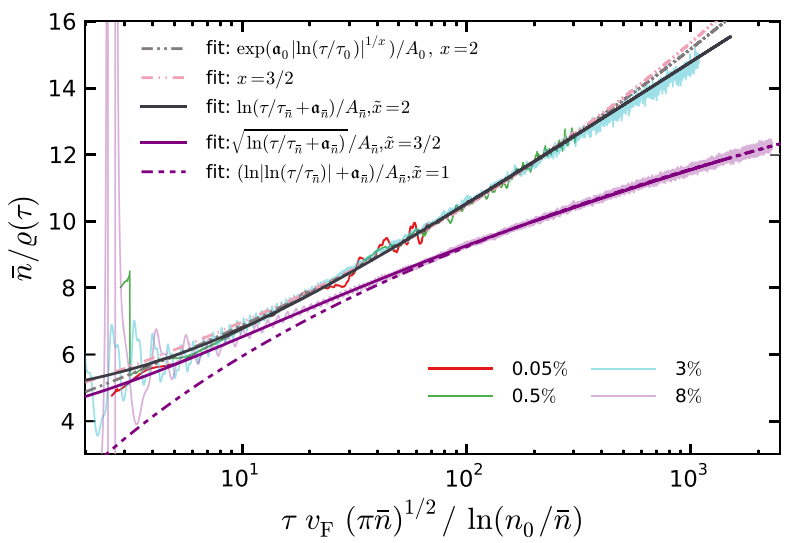

FIG. 2 (color online). Data collapse of the inverse time series on a master curve for different vacancy concentrations: $\bar{n}=0.05 \%, 0.5 \%, 3 \%$. It is seen that at preasymptotic times $1 \ll \mathfrak{D} \tau \ll \mathfrak{D} \tau_{\bar{n}}^{*}$, Gade-Wegner scaling, Eq. (1), works well within the simulation time window. However, at very long times $\tau \gg \tau_{\bar{n}}^{*}$, the increase of $\bar{n} / \varrho(\tau)$ becomes sublinear so that GadeWegner scaling is violated. This time regime is numerically accessible at vacancy concentration $\bar{n} \gtrsim 8 \%$. Motivated by Ref. [23], we fit $\sqrt{\ln \left(\tau / \tau_{0}+\mathfrak{a}_{0}\right)} / A_{0}, \tilde{x}=3 / 2$, but alternatives [dashed lines; $\ln (\tau), \tilde{x}=2$ and $\ln \ln (\tau), \tilde{x}=1$ ] are also shown for comparison. The fluctuations in the raw data reflect the stochastic nature of the methodology. (Fitting parameters for fits shown here and in all subsequent figures are listed in the Supplemental Material [24].)

indicates the existence of a second plateau in the limit $\xi \rightarrow \infty$ that exists at $q_{c} \leq q<1$ and that is not yet fully developed in our data. The plateau is a manifestation of the fact that certain moments, $q>q_{c}$, also of the critical wave functions become insensitive to the system size growth and are (in this sense) frozen.

Collapsing the IPRs on the master curve, Fig. 4, delivers $\tau_{q}$ and $\xi(E)$ in units of $\xi_{0} \equiv \xi\left(E_{0}\right)$ for a reference energy $E_{0}$. The multifractal spectrum $\tau_{q}$ is displayed in Fig. 4,

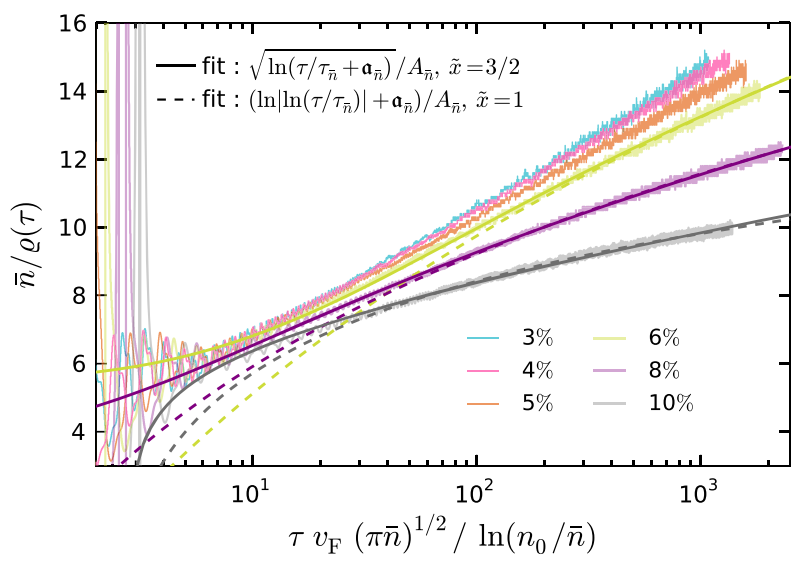

FIG. 3 (color online). Evolution of $\bar{n} / \varrho(\tau)$ at larger vacancy concentrations $(\bar{n}=3 \%-10 \%)$ where very long (effective) times $\tau \gg \tau_{\bar{n}}$ fall within our simulation window. Fits correspond to $\tilde{x}=3 / 2$ (solid lines) and $\tilde{x}=1$ (dashed lines), defined in Eq. (6). 


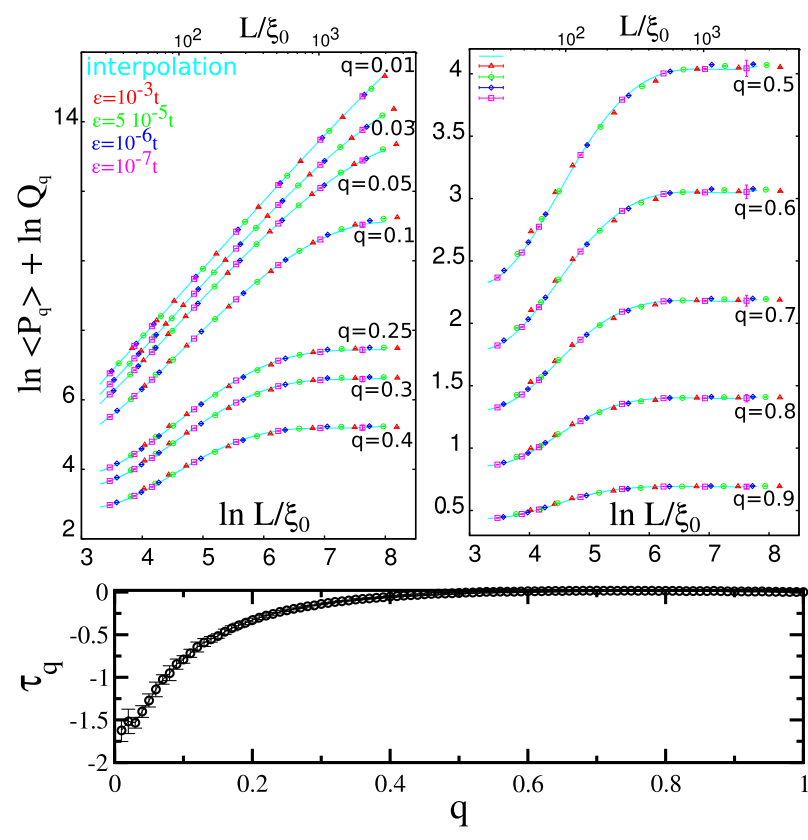

FIG. 4 (color online). Top panels: master curves for different $q$ values as obtained after rescaling of $x, y$ axes with energydependent scale factors $\xi(E)$ and $Q_{q}=\xi(E)^{\tau_{q}}$ (not shown). Parameters: $\bar{n}=4 \%, L=64-2048, \epsilon=10^{-3}, 5 \times 10^{-5}, 10^{-6}$, $10^{-7}$. IPR-distribution functions are given in the Supplemental Material [24]. Bottom panel: multifractal spectrum as estimated from fitting to $Q_{q}$; it displays frozen multifractality.

lower panel. It supports the freezing scenario and gives a rough estimate of $q_{c} \lesssim 0.5$. The localization length is shown in Fig. 5 and compared with the DoS data converted into $\xi(E)$ via Eq. (3). (By matching both $\xi$ traces at $\epsilon=10^{-3} t$ we fix the scale $\xi_{0}$.) The result is satisfactory in the sense that the matching procedure smoothly interpolates from the high-energy (SCTMA) into the very-low energy regime. This trace summarizes our second key statement. Namely, a consistent fit is achieved with $\tilde{y}=1$ and $\tilde{x}=3 / 2$ over data spanning more than 5 orders of magnitude in energy. This result is in full agreement with the prediction by Ostrovsky et al. [23].

Conclusions.-General implications: A first important conclusion from our numerical study is that the canonical $\sigma$ model of symmetry class BDI does not apply to the case of graphene with vacancies. One expects that the underlying reason is related to the fact that vacancies in the tightbinding representation should be understood as very strong ("unitary") scatterers that enforce zero amplitude of the scattering wave function at the scattering center. In this sense, the individual scatterer is never weak, which is at odds with the assumption underlying the derivation of the generic $\sigma$ model underlying the Altland-Zirnbauer classification.

In principle, the observation that not only the symmetry class (here BDI) but also the type of disorder plays a crucial role in determining the low-energy behavior has been made

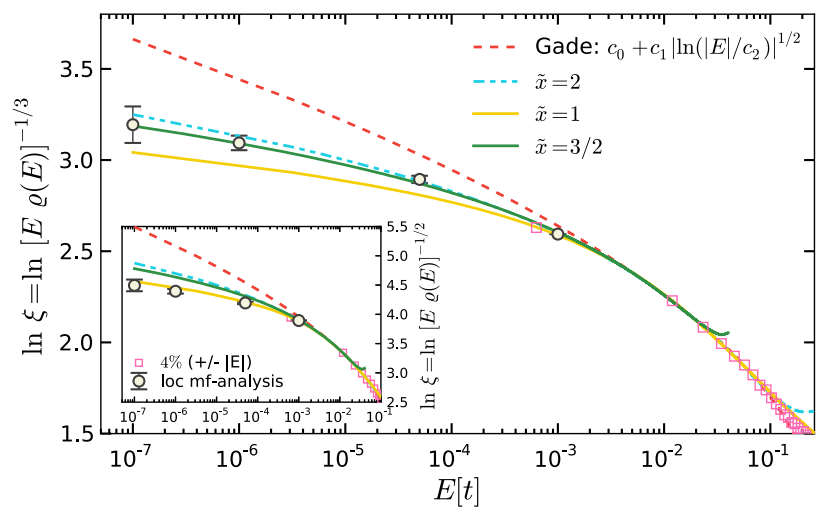

FIG. 5 (color online). Localization length as obtained from the DoS (triangles, data Fig. 1) and from the generalized multifractal analysis (symbols) at vacancy concentration $4 \%$. The conversion of the DoS has been done following Eq. (3) assuming $\tilde{y}=1$. Four fits display Fourier transformation of Eq. (5) (red dashed line, Gade-Wegner-form) and Eq. (6) (blue dot-dashed line: $\tilde{x}=2$; green solid line: $\tilde{x}=3 / 2$; yellow line: $\tilde{x}=1$ ). As seen here, Eq. (6) with $\tilde{x}=3 / 2$ fits all regimes best (using three fitting parameters). Inset: Conversion of DoS into $\xi(E)$ assuming $\tilde{y}=0$. Comparison illustrates that $\tilde{y}$, indeed, enters the data interpretation in an important way, since for $\tilde{y}=0$ only $\tilde{x}=1$ would provide an acceptable fit.

before [35]. Of particular interest here are disordered $d$-wave superconductors with very strong scatterers. They belong to chiral class AIII, which is the unitary cousin of BDI. Its $\sigma$ model also exhibits the Gade singularity, Eq. (1) [36]. In this context, an interesting proposal deviating from the Gade-Wegner form has been made [18,37] [e.g., $\varrho(E) \sim 1 /\left|E \ln (E)^{2}\right|$, i.e., $\tilde{x}=2$ in our nomenclature], but so far its status has been controversial [38]. In a recent study [40], a very similar model, the Kitaev model that has a representation in terms of a bipartite random hopping problem of Majorana fermions on a hexagonal lattice in the background of $Z_{2}$ fluxes, was shown to have a similar singular DoS with $\tilde{x} \approx 1.7$. However, these results were obtained in the gapped phase of the model, wherein the isolated impurity states are exponentially localized -as opposed to a $1 / r$ envelop of vacancy-induced zero modes in graphene. Hence, the relation of this result to graphene with vacancies is uncertain.

Microscopic realizations and graphene: From the point of view of graphene research, the relevance of our results depends on the applicability of the approximation of disorder as an ensemble of unitary scatters. Such unitary scatterers are realized at least approximately, e.g., when a carbon atom forms a chemical bond with an absorbate and, therefore, is taken from the $s p 2$ into the $s p 3$ hybridization. Indeed, an isolated $s p 3$ hybrid induces a state typically of the order of $10 \mathrm{meV}$ away from midgap [5]. The zero mode of the tight-binding vacancy should be understood as an approximation for such a state. Correspondingly, we might expect that the structure of the DoS that we study here 
could be representative for the real material on the scale of several meV, i.e., well above $10^{-3} t$. Hence, the intermediate energy window, which displays the quantum interference enhanced increase of the DoS, should still be experimentally accessible, but the ultralow energy range might prove difficult to reach.

We thank J. Chalker, I. Gornyi, A. Mirlin, Chr. Mudry, H. Obuse, and P. Ostrovsky for helpful discussions. Especially, we express our gratitude to I. Gornyi and P. Ostrovsky for sharing analytical results with us prior to publication. We acknowledge the DFG (CFN and SPP 1243 ) for financial support. We also thank I. Kondov and the Jülich Supercomputer Center (JUROPA, project HKA12) for computational assistance and resources. R. N. and S. B. acknowledge the use of the HPC facility of IITMadras.

Corresponding author. soumya.bera@neel.cnrs.fr

[1] A. H. Castro Neto, F. Guinea, N. M. R. Peres, K. S. Novoselov, and A. K. Geim, Rev. Mod. Phys. 81, 109 (2009).

[2] V. M. Pereira, F. Guinea, J. M. B. Lopes dos Santos, N. M. R. Peres, and A. H. Castro Neto, Phys. Rev. Lett. 96, 036801 (2006).

[3] V. M. Pereira, J. M. B. Lopes dos Santos, and A. H. Castro Neto, Phys. Rev. B 77, 115109 (2008).

[4] D. A. Abanin, A. V. Shytov, and L. S. Levitov, Phys. Rev. Lett. 105, 086802 (2010).

[5] T. O. Wehling, S. Yuan, A. I. Lichtenstein, A. K. Geim, and M. I. Katsnelson, Phys. Rev. Lett. 105, 056802 (2010).

[6] A. Altland and M. R. Zirnbauer, Phys. Rev. B 55, 1142 (1997).

[7] F. Evers and A. D. Mirlin, Rev. Mod. Phys. 80, 1355 (2008).

[8] R. Gade and F. Wegner, Nucl. Phys. B360, 213 (1991).

[9] R. Gade, Nucl. Phys. B398, 499 (1993).

[10] The result was confirmed by Guruswamy et al. [11] in their analysis of a bipartite $\pi$-flux model that also belongs to class BDI. Specifically, this study shows that generic representatives of BDI exhibit a running coupling $g_{A} \sim \ln L$. Within the $\pi$-flux model $g_{A}$ has the interpretation of a coupling to a real random gauge field. Since one has $z \approx 1+2 g_{A}$, the previous conclusion $z(L) \sim \ln L$ is a consequence of "runaway flow."

[11] S. Guruswamy, A. LeClair, and A. W. W. Ludwig, Nucl. Phys. B583, 475 (2000).

[12] O. Motrunich, K. Damle, and D. A. Huse, Phys. Rev. B 65, 064206 (2002).

[13] C. Mudry, S. Ryu, and A. Furusaki, Phys. Rev. B 67, 064202 (2003).

[14] S. Yuan, H. De Raedt, and M. I. Katsnelson, Phys. Rev. B 82, 115448 (2010).

[15] T. Stauber, N. M. R. Peres, and A. H. Castro Neto, Phys. Rev. B 78, 085418 (2008).

[16] S. Wu, L. Jing, Q. Li, Q. W. Shi, J. Chen, H. Su, X. Wang, and J. Yang, Phys. Rev. B 77, 195411 (2008).
[17] P. M. Ostrovsky, I. V. Gornyi, and A. D. Mirlin, Phys. Rev. B 74, 235443 (2006).

[18] C. Chamon and C. Mudry, Phys. Rev. B 63, 100503 (2001).

[19] P. J. Hirschfeld and W. A. Atkinson, J. Low Temp. Phys. 126, 881 (2002).

[20] At our system sizes, $L=2048$, 4096, we did not observe a significant effect of $\phi$ averaging on $\varrho(E)$ due to selfaveraging. If not specified otherwise, averaging was over four initial states [21,22]. A convergence test justifying the choice of $d t$ is given in the Supplemental Material [24].

[21] V. Häfner, Diploma Thesis, Karlsruhe Institute of Technology, 2011.

[22] J. Schindler, Master's Thesis, Karlsruhe Institute of Technology, 2012.

[23] P. M. Ostrovsky, I. V. Protopopov, E. J. König, I. V. Gornyi, A. D. Mirlin, and M. A. Skvortsov, Phys. Rev. Lett. 113, 186803 (2014).

[24] See the Supplemental Material at http://link.aps.org/ supplemental/10.1103/PhysRevLett.113.186802, which includes Refs. [4,23,25-30].

[25] G. D. Mahan, Many-Particle Physics (Springer, New York, 2000).

[26] N. Weik, Diploma Thesis, Karlsruhe Institute of Technology, 2013.

[27] T. Ando, Y. Zheng, and H. Suzuura, J. Phys. Soc. Jpn. 71, 1318 (2002).

[28] P. M. Ostrovsky, I. V. Gornyi, and A. D. Mirlin, Phys. Rev. B 74, 235443 (2006).

[29] B. Yu-Kuang Hu, E. H. Hwang, and S. Das Sarma, Phys. Rev. B 78, 165411 (2008).

[30] A. Altland and B. Simons, Condensed Matter Field Theory (Cambridge University Press, Cambridge, England, 2006).

[31] A. Rodriguez, L. J. Vasquez, K. Slevin, and R. A. Römer, Phys. Rev. Lett. 105, 046403 (2010).

[32] F. Evers, A. Mildenberger, and A. D. Mirlin, Phys. Rev. B 64, 241303(R) (2001).

[33] A. R. Subramaniam, I. A. Gruzberg, A. W. W. Ludwig, F. Evers, A. Mildenberger, and A. D. Mirlin, Phys. Rev. Lett. 96, 126802 (2006).

[34] R. B. Lehoucq, D. C. Sorensen, and C. Yang, Arpack Users Guide: Solution of Large Scale Eigenvalue Problems by Implicitly Restarted Arnoldi Methods (SIAM, Philadelphia, 1998).

[35] For instance, symmetry class D: It allows for different lattice models (e.g., of the Chalker-Coddington type) that exhibit very different phase diagrams depending on the form of the disorder. Some may exhibit only (topologically different) localized phases (RBIM), but others may also support metallic phases (Cho-Fisher model). For a review, see Ref. [7].

[36] A. Altland, Phys. Rev. B 65, 104525 (2002).

[37] C. Pépin and P. A. Lee, Phys. Rev. B 63, 054502 (2001).

[38] For an overview, see e.g., Refs. [19,39].

[39] A. V. Balatsky, I. Vekhter, and J.-X. Zhu, Rev. Mod. Phys. 78, 373 (2006).

[40] A. J. Willans, J. T. Chalker, and R. Moessner, Phys. Rev. B 84, 115146 (2011). 